\title{
Mcm2 phosphorylation and the response to replicative stress
}

\author{
Brent E Stead, Christopher J Brandl, Matthew K Sandre and Megan J Davey*
}

\begin{abstract}
Background: The replicative helicase in eukaryotic cells is comprised of minichromosome maintenance (Mcm) proteins 2 through 7 (Mcm2-7) and is a key target for regulation of cell proliferation. In addition, it is regulated in response to replicative stress. One of the protein kinases that targets Mcm2-7 is the Dbf4-dependent kinase Cdc7 (DDK). In a previous study, we showed that alanine mutations of the DDK phosphorylation sites at S164 and S170 in Saccharomyces cerevisiae Mcm2 result in sensitivity to caffeine and methyl methanesulfonate (MMS) leading us to suggest that DDK phosphorylation of Mcm2 is required in response to replicative stress.
\end{abstract}

Results: We show here that a strain with the $m \mathrm{~cm} 2$ allele lacking DDK phosphorylation sites $\left(m c m 2_{A A}\right)$ is also sensitive to the ribonucleotide reductase inhibitor, hydroxyurea (HU) and to the base analogue 5-fluorouracil (5-FU) but not the radiomimetic drug, phleomycin. We screened the budding yeast non-essential deletion collection for synthetic lethal interactions with $m \mathrm{~cm} 2_{A A}$ and isolated deletions that include genes involved in the control of genome integrity and oxidative stress. In addition, the spontaneous mutation rate, as measured by mutations in CAN1, was increased in the $m c m 2_{A A}$ strain compared to wild type, whereas with a phosphomimetic allele $\left(m c m 2_{E E}\right)$ the mutation rate was decreased. These results led to the idea that the $m \mathrm{~cm}{ }_{\mathrm{AA}}$ strain is unable to respond properly to DNA damage. We examined this by screening the deletion collection for suppressors of the caffeine sensitivity of $m \mathrm{~cm} 2_{A A}$. Deletions that decrease spontaneous DNA damage, increase homologous recombination or slow replication forks were isolated. Many of the suppressors of caffeine sensitivity suppressed other phenotypes of $m \mathrm{~cm} 2_{A A}$ including sensitivity to genotoxic drugs, the increased frequency of cells with RPA foci and the increased mutation rate.

Conclusions: Together these observations point to a role for DDK-mediated phosphorylation of Mcm2 in the response to replicative stress, including some forms of DNA damage. We suggest that phosphorylation of Mcm2 modulates Mcm2-7 activity resulting in the stabilization of replication forks in response to replicative stress.

\section{Background}

DNA replication is tightly regulated to ensure that genomes are copied once and only once per cell division cycle [1]. In addition, cells must respond to assaults that damage DNA and/or disrupt replication forks by preventing the initiation of DNA replication and stabilizing active replication forks [2]. One of the targets for these regulatory events is the replicative helicase that unwinds DNA at the replication fork [3-8]. The catalytic core of the replicative helicase in eukaryotic cells is a heterohexameric complex comprised of the minichromosome maintenance proteins 2 through 7 (Mcm2-7; $[9,10])$. Mcm2-7 activity is tightly

\footnotetext{
* Correspondence: mdavey5@uwo.ca

Department of Biochemistry, Schulich School of Medicine \& Dentistry, University of Western Ontario, London, ON N6A 5C1, Canada
}

controlled during the initiation of DNA replication and is targeted in response to replicative stress [3,6-8,11-14]. In vivo, Mcm2-7 functions within the CMG complex comprised of Cdc45, Mcm2-7 and the tetrameric GINS complex (Sld5, Psf1, Psf2 and Psf3) [11,12]. The catalytic activity for DNA unwinding resides in $\mathrm{Mcm} 2-7$ with Cdc45 and GINS playing roles in limiting Mcm2-7 activity to $S$ phase and providing scaffolding functions within the replisome [11,15-17]. CMG is isolated from replicating yeast cells as part of the RPC (replisome progression complex) that includes the checkpoint protein Mrc1, the fork pausing complex Tof1-Csm3, the histone chaperone FACT and the sister chromatid cohesion factor, Ctf4 [16].

Mcm2-7 activity is also regulated by phosphorylation. A recent study from our laboratory showed that 
phosphorylation of Saccharomyces cerevisiae Mcm2 by the Dbf4-dependent kinase, Cdc7 (DDK) at S164 and S170 is important for a proper response to DNA damage [5]. Strains containing a non-phosphorylatable allele of $m c m 2\left(m c m 2_{A A}\right)$ grow similarly to wild type cells in normal growth conditions but are sensitive to the DNA alkylating agent, methyl methanesulfonate (MMS) and to caffeine. Caffeine is a purine analogue with pleiotropic effects. In general, caffeine inhibits PI3K-related kinases, which in yeast include TOR (Tor1 and Tor2), Mec1 and Tel1 [18-21]. TOR controls cell growth in response to nutrients and stress whereas Mec1 and Tel1 are both checkpoint kinases that also have roles in control of replication initiation (Mec1) and telomere maintenance (Tel1) [3,22-24].

Here, we show that in addition to MMS and caffeine, the $m c m 2_{A A}$ strain is sensitive to the ribonucleotide reductase inhibitor, hydroxyurea $(\mathrm{HU})$ and the base analogue 5fluorouracil (5-FU), but not phleomycin, a radiomimetic drug. The phosphomimetic glutamic acid substitutions at S164 and S170 suppress sensitivity to these drugs. We examined the genetic network within which $m c m 2_{A A}$ functions and found 9 deletions that have synthetic slow growth or lethal interactions with $m c m 2_{A A}$ and 16 deletions that suppress the caffeine sensitivity of $m c m 2_{A A}$. The identities of these gene deletions are consistent with a role for $\mathrm{Mcm} 2$ phosphorylation in the response to DNA damage and replicative stress and include two members of the RPC. A role in response to replicative stress is emphasized by the higher than wild type spontaneous mutation rate in the $m c m 2_{A A}$ strain and a lower than wild type mutation rate with the $m c m 2_{E E}$ phosphomimetic allele. Most of the gene deletions that suppressed the caffeine sensitivity of $m c m 2_{A A}$ also relieved other phenotypes of $m c m 2_{A A}$. We propose that phosphorylation of $\mathrm{Mcm} 2$ by DDK is required in response to replicative stress to stabilize Mcm2-7 at replication forks.

\section{Results}

\section{Growth of $m c m 2_{A A}$ and $m c m 2_{E E}$ cells in the presence of} replicative stress

We examined the growth of the $m c m 2_{A A}$ strain on media containing agents that cause replicative stress (Figure 1A \& B). The $m c m 2_{A A}$ strain had reduced growth relative to $M C M 2$ on YPD plates containing MMS or 5-FU (Figure 1A), but not on plates containing phleomycin (Figure 1B). These agents have different effects on DNA stability in budding yeast. MMS damages DNA by methylating guanines and adenines [25]. The effects of 5-fluorouracil in yeast are twofold: it inhibits the pyrimidine biosynthesis pathway and results in misincorporation of uracil into nascent DNA [26]. Phleomycin is structurally similar to bleomycin, a radiomimetic drug that causes double stranded DNA breaks [27]. The $m c m 2_{A A}$ strain also grows poorly upon constant exposure to the ribonucleotide reductase inhibitor, hydroxyurea $(\mathrm{HU})$, which interferes with the integrity of DNA replication forks and induces an $\mathrm{S}$ phase checkpoint $[28,29]$.

We noted previously that Mcm2 in which S164 and S170 are altered to glutamic acids $\left(m c m 2_{E E}\right)$ acts like a phosphomimic, allowing growth of cells in the presence of caffeine and MMS, and has the same activity in vitro as phospho- $\mathrm{Mcm} 2$ [5]. If phosphorylation of $\mathrm{Mcm} 2$ is required in response to 5 -FU and $\mathrm{HU}$, then $m c m 2_{E E}$ should be insensitive to these agents. As predicted, the $m c m 2_{E E}$ strain grew similarly to wild type cells in the presence of 5-FU and HU (Figure 1C). Substitution of Glu for S at position 164 or 170 also resulted in wild type growth consistent with the requirement to mutate both Ser to Ala to obtain a phenotype [5].

\section{Synthetic lethal/slow growth interactions with $\mathrm{mcm} 2_{A A}$}

The sensitivity of the $m c m 2_{A A}$ strain to caffeine, MMS, 5-FU and $\mathrm{HU}$ suggests that phosphorylation of $\mathrm{Mcm} 2$ is required in response to replicative stress. Furthermore, the increased frequency of RPA foci in these cells [5] suggests disruption of replication forks or an inability to respond to replicative stress [30-32]. If our model is correct, then mutations that increase genomic instability will be synthetic lethal or show slow growth with $m c m 2_{A A}$. After screening the $S$. cerevisiae non-essential deletion collection for synthetic lethal interactions with $m c m 2_{A A}$ and confirming the interactions by tetrad dissection, we found 8 gene deletions that result in no or slow growth when combined with $m c m 2_{A A}$ (Table 1 and Additional file 1). Three of the eight gene deletions that display synthetic lethal or slow growth interactions with $m c m 2_{A A}$ affect cell stress responses or cell cycle. In particular, the synthetic interaction of $m c m 2_{A A}$ with $\operatorname{chk} 1 \Delta$, a deletion in the gene encoding a checkpoint effector kinase is consistent with the idea that $m c m 2_{A A}$ is important in response to replicative stress. In addition, $c t f 4 \Delta$, $\operatorname{sod} 1 \Delta$ and img1s all lead to genomic instability or increase DNA damage [33-36] and their negative synthetic interactions with $m c m 2_{A A}$ support the idea that Mcm2 phosphorylation is important in response to DNA damage.

\section{Rad53 is phosphorylated in the $m c m 2_{A A}$ strain}

One potential role of $\mathrm{Mcm} 2$ phosphorylation in response to replicative stress is in the induction of a checkpoint signal leading to phosphorylation of Rad53, detected by decreased migration through SDS-PAGE. We examined Rad53 by Western blotting in the $M C M 2$ and $m c m 2_{A A}$ strains before and after treatment with 0.02\% MMS, which triggers the $\mathrm{S}$ phase checkpoint. As seen in Figure 2, the migration of Rad53 is slower in the presence of MMS in both strains, suggesting that signalling in response to DNA 


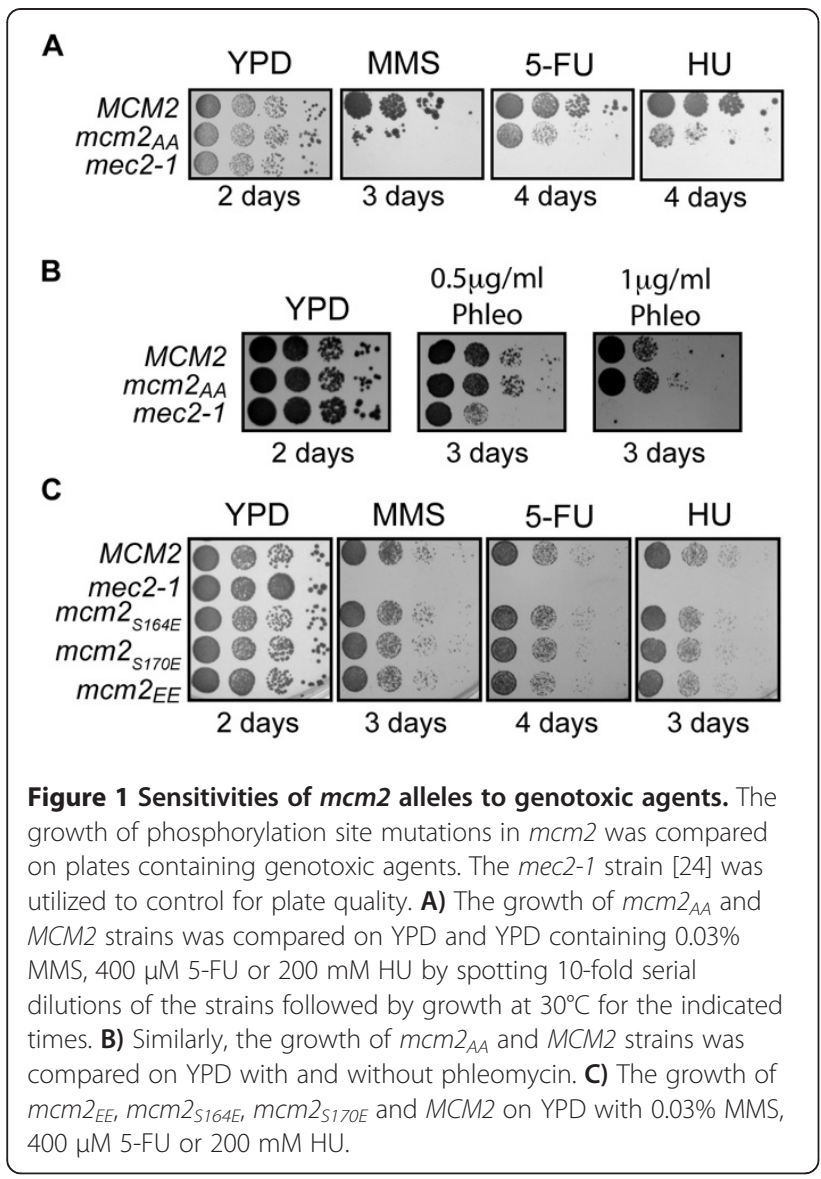

damage is intact and that phosphorylation of $\mathrm{Mcm} 2$ is not required to activate checkpoint. We also tested for a genetic interaction between $m c m 2_{A A}$ and a checkpoint deficient allele of RAD53 (mec2-1 [24]). After mating the strains and generating spore progeny by tetrad dissection,

Table 1 Synthetic lethal or slow growth interactions with mcm $2_{A A}$

\begin{tabular}{lllll}
\hline Gene & ORF & \multicolumn{1}{c}{ Function } & GO & growth \\
\hline chk1 $\Delta$ & YBR274W & Checkpoint Kinase & $1,2,5$ & lethal \\
\hline ctf4 $\Delta$ & YPR135W & Sister Chromatid Cohesion & 1,2 & lethal \\
\hline sod1 $\Delta$ & YJR104C & Response to oxygen radicals & $1,6,8$ & slow \\
\hline bud23 $\Delta$ & YCR047C & Bud site selection & $2,3,4$ & lethal \\
\hline pep3 $\Delta$ & YLR148W & $\begin{array}{l}\text { Vesicular docking/Nacuolar } \\
\text { biogenesis }\end{array}$ & 3,8 & lethal \\
\hline skn1 $\Delta$ & YGR143W & Sphingolipid biosynthesis & 7,8 & slow \\
\hline img1 & YCR046C & $\begin{array}{l}\text { Mitochondrial genome } \\
\text { maintenance }\end{array}$ & 9 & lethal \\
\hline vma13 & YPR036W & Subunit of Vacuolar ATPase & 10 & lethal
\end{tabular}

Genes are grouped by their GO terms as annotated in the Saccharomyces cerevisiae database [37]. Gene ontology: (1) Response to cell stress/chemical stimuli (2) cell cycle, (3) transport, (4) RNA metabolic process, (5) signalling process/protein modification process, (6) transcription, (7) carbohydrate metabolic process, (8) cell wall, membrane, \& vesicle mediated transport, (9) mitochondrial organization, and (10) other.

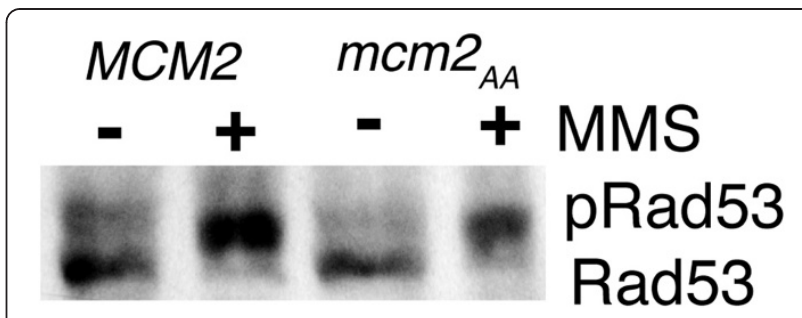

Figure 2 Rad53 activation in the $m \mathrm{~cm} 2_{A A}$ strain. Western blots of crude protein lysates of $m c m 2_{A A}$ or $M C M 2$ strains probed with antiRad53 antibody before and after treatment with MMS. Log phase cultures in YPD were grown for 2 hours at $30^{\circ} \mathrm{C}$ in the presence or absence of $0.02 \%$ MMS. After harvesting by centrifugation, protein was extracted with TCA, as described [38]. Approximately $20 \mu \mathrm{g}$ of total protein was examined by Western blotting using anti-Rad53 antibody (Santa Cruz Biotechnology, SC-6749) as primary antibody followed by rabbit anti-goat lgG coupled to horseradish peroxidase (Sigma-Aldrich). The blots were visualised using the Supersignal West Pico chemiluminescence kit (Pierce) and x-ray film.

none of the spore colonies contained both mutations indicating a synthetic lethal interaction, consistent with $\mathrm{Mcm} 2$ phosphorylation functioning in a parallel pathway to Rad53 (Additional file 1).

\section{Increased mutation rate in $m \mathrm{~cm} 2_{A A}$ cells}

If phosphorylation of $\mathrm{Mcm} 2$ is important in response to DNA damage and/or replicative stress, cells containing the non-phosphorylatable allele of $m c m 2\left(m c m 2_{A A}\right)$ would be predicted to accumulate mutations at a higher rate than cells with $M C M 2$ or $m c m 2_{E E}$. To test this, we utilized the CAN1 forward mutation assay in which a mutation rate is determined from the number of canavanine resistant colonies that arise. CAN1 encodes a transporter that enables the toxic compound canavanine to enter cells. If CAN1 function is lost, then the cell is rendered resistant to canavanine. We grew CAN1 strains containing different alleles of $m c m 2$ for several generations in liquid media without selection before determining the number of canavanine resistant colonies and the mutation rate using the method of the median $[39,40]$. The mutation rate was nearly two-fold higher in the $m c m 2_{A A}$ strain than with $M C M 2\left(5.5 \times 10^{-7}\right.$ v. $\left.3.2 \times 10^{-7}\right)$, consistent with an inability to respond properly to spontaneous DNA damage in the absence of $\mathrm{Mcm} 2$ phosphorylation. Significantly, the mutation rate in the $m c m 2_{E E}$ strain was half that of the MCM2 strain $\left(1.6 \times 10^{-7}\right)$.

\section{Suppressors of the caffeine sensitivity of $m \mathrm{~cm} 2_{A A}$}

As $m c m 2_{A A}$ is predicted to interfere in the response to replicative stress, second site mutations that decrease DNA damage or increase the capacity for DNA repair would be expected to act as suppressors. We therefore screened the haploid deletion strain collection for gene deletions that suppress the caffeine sensitivity of $m c m 2_{A A}$. 
Sensitivity to caffeine was chosen because of the strong phenotype it elicits with $m c m 2_{A A}$. Candidates were re-mated, isolated by tetrad dissection and re-tested on YPD with caffeine. Sixteen gene deletions were identified (Figure 3). We classified these genes by biological functions based on gene ontology annotations in the Saccharomyces Genome Database [37] as well as their reported functions in the literature (Table 2). These classifications yielded four groups of genes: cell stress, cell cycle, protein folding and "other" functions. Interestingly, half of the deletions, when independent of $m c m 2_{A A}$, were sensitive to caffeine indicating roles for these genes in response to caffeine. Four of these, tof $1 \Delta, \operatorname{mbp} 1 \Delta$, ume $6 \Delta$ and sip $18 \Delta$ were as sensitive to caffeine as $m c m 2_{A A}$. Others, such as $\operatorname{rad} 9 \Delta$, $r a d 2 \Delta$, $p d r 15 \Delta$ and $h r d 1 \Delta$, displayed an intermediate sensitivity. In addition, three showed decreased sensitivity to caffeine compared to wild type MCM2 (yhp1A, $s s m 4 \Delta$ and rplsb 4 ). We also note that some of the deletions, such as tof $1 \Delta$, rad $9 \Delta$ and pac10 , resulted in only partial suppression (Figure 3).

Our hypothesis predicts that deletions that suppress the caffeine sensitivity of $m c m 2_{A A}$ will also decrease the mutation rate in the $m c m 2_{A A}$ strain. Therefore, we repeated the CAN1 forward mutation assay on a subset of the deletion strains. As shown in Figure 4, seven of the 11 deletions tested decreased the mutation rate, both with the deletion alone and in the presence of $m c m 2_{A A}$. The exceptions were tof $1 \Delta, \operatorname{rad} 2 \Delta$ and $u m e 6 \Delta$. Deletion of tof1 causes genomic instability [41-43] and $\mathrm{rad} 2 \Delta$ is deficient in nucleotide excision repair [44]. Ume6 is involved in the expression of several genes and deletion of ume6 increases homologous recombination [45-48].

To further evaluate the mechanisms by which the gene deletions suppress $m c m 2_{A A}$, we spotted strains containing the suppressor deletions and either $M C M 2$ or $m c m 2_{A A}$ onto YPD plates containing MMS, 5-FU and HU (Figure 5, Table 3 and Additional file 1). All of the deletions that suppressed the caffeine sensitivity of $m c m 2_{A A}$ also suppressed at least one other drug sensitivity of $m \mathrm{~cm} 2_{A A}$, exemplified by $\operatorname{rad} 2 \Delta$ and $\operatorname{ssm} 4 \Delta$ (Figure 5). Many of the deletions in the cell stress group also lead to sensitivity to these drugs in the MCM2 background, thus likely accounting for the complex phenotypic patterns. For example, the srs 2 and rad 9 deletions do not suppress the sensitivity of $m c m 2_{A A}$ to MMS or 5-FU and only partially suppress on $\mathrm{HU}$ (Figure 5). Some of the deletions decrease sensitivity to the drugs in an otherwise wild type background. For example, the $y$ hp1 $1 \Delta$ strain grows faster than the wild type strain on plates containing MMS or 5-FU (Figure 5). This increased growth is also noted in the $m c m 2_{A A} y h p 1 \Delta$ strain. Therefore, the $y h p 1$ deletion likely functions non-specifically to suppress $m c m 2_{A A}$. Of note, Yhp1 is a transcriptional repressor that along with Yox1 is involved in the cyclic transcription of a set of genes that includes MCM2-7 [49].

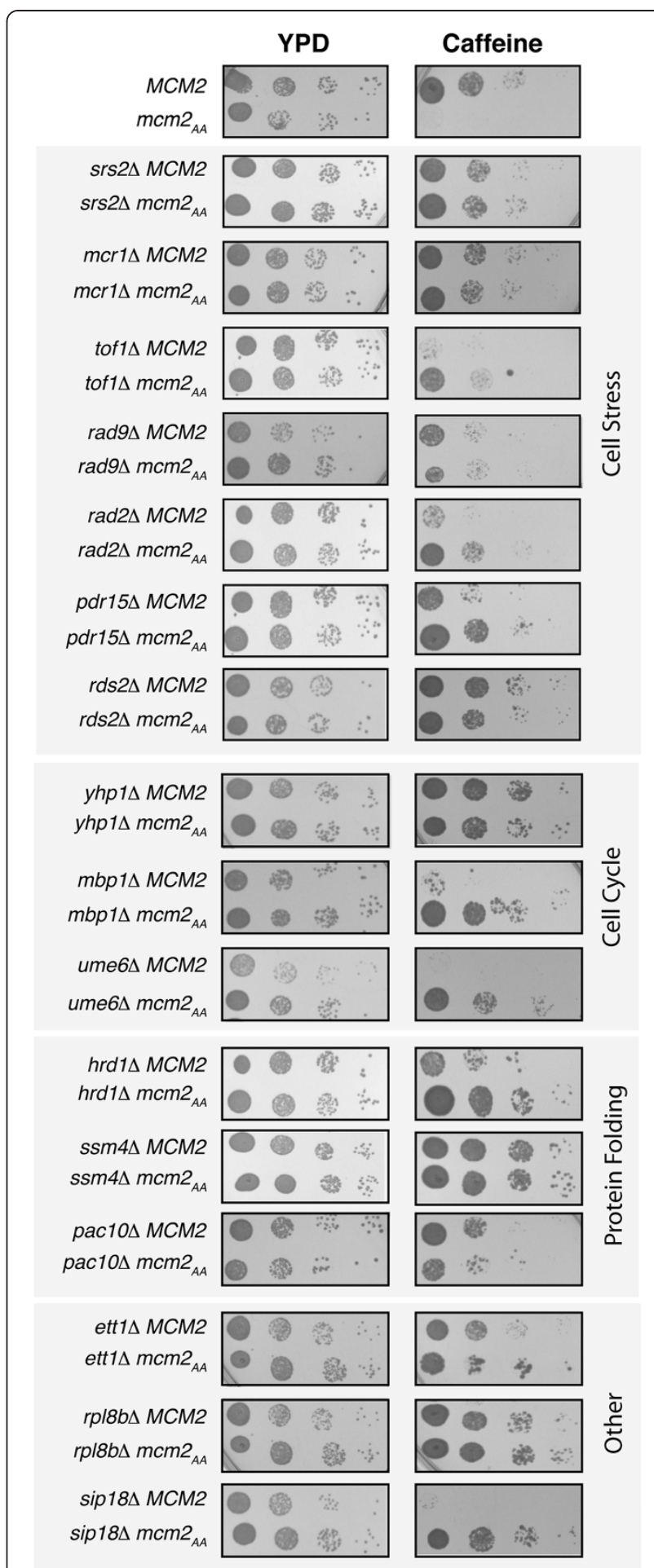

Figure 3 Identification of gene deletions that suppress $m c m 2_{A A}$. The growth of strains with deletions that suppress the caffeine sensitivity of $m c m 2_{A A}$ was compared on YPD and YPD with $15 \mathrm{mM}$ caffeine. Approximately 5000 cells $/ \mathrm{ml}$ and 10 -fold serial dilutions were spotted and grown at $30^{\circ} \mathrm{C}$. 
Table 2 Suppressors of the caffeine sensitivity of $\mathbf{m c m} \mathbf{2}_{A A}$

\begin{tabular}{llll}
\hline Gene & \multicolumn{1}{c}{ ORF } & \multicolumn{1}{c}{ Function } & GO \\
\hline srs2 $\Delta$ & YJL092C & DNA repair, helicase & 1 \\
\hline mcr1 $\Delta$ & YKL150W & Oxidative stress response & 1 \\
\hline tof1 $\Delta$ & YNL273W & Subunit of fork pausing complex & $1,2,5$ \\
\hline rad9 $\Delta$ & YDR217C & Transmission of checkpoint signal & $1,2,4,56$ \\
\hline rad2 $\Delta$ & YGR258C & Nucleotide excision repair & $1,4,6$ \\
\hline pdr15 $\Delta$ & YDR406W & Cellular detoxification & 1,3 \\
\hline rds2 $\Delta$ & YPL133C & Transcription factor & $1,6,7$ \\
\hline yhp1 $\Delta$ & YDR451C & Transcription factor/cell cycle & $2,4,6$ \\
\hline mbp1 $\Delta$ & YDL056W & Transcription factor/cell cycle & $2,4,6$ \\
\hline ume6 $\Delta$ & YDR207C & Transcription factor & $2,4,5,6$ \\
\hline hrd1 $\Delta$ & YOL013C & Ubiquitin ligase/ER assoc. decay & 8 \\
\hline ssm4 $\Delta$ & YIL030C & Ubiquitin ligase/ER assoc. decay & 8 \\
\hline pac10 $\Delta$ & YGR078C & Protein folding & 8 \\
\hline sip18 $\Delta$ & YMR175W & Osmotic stress & 9 \\
\hline rpl8b $\Delta$ & YLL045C & Ribosomal protein & 9 \\
\hline ett1 $\Delta$ & YOR051C & Translation termination & 9 \\
\hline
\end{tabular}

Genes are grouped by their GO terms as annotated in the Saccharomyces cerevisiae database [37]. Horizontal lines separate different classes. Gene ontology: (1) Response to cell stress/chemical stimuli (2) cell cycle, (3) transport, (4) RNA metabolic process, (5) signalling process/protein modification process, (6) transcription, (7) carbohydrate metabolic process, (8) ER-mediated degradation \& protein-folding and (9) other.

However, deletion of $y h p 1$ alone did not affect expression of MCM3-lacZ and had little or no effect on cell growth [49]. The ett1 $\Delta$ deletion on MMS or HU and sip18A on HU similarly increase growth of cells containing the wild type and mutated $m c m 2$ alleles (Table 3 and Additional file 1).

Previously, we observed that the $m c m 2_{A A}$ strain has a higher frequency of cells with RPA foci [5]. RPA is the single-stranded DNA binding protein and thus foci represent generation of single stranded DNA. In wild type cells, RPA is diffuse in the nucleus (Figure 6A). In a low percentage of wild type cells, RPA foci will appear. Since a higher frequency of cells contain foci when treated with DNA damaging agents, the foci are thought to represent ongoing repair processes or disruption of the replication fork, both of which generate stretches of single stranded DNA [30-32]. Interestingly, in cells containing $m c m 2_{A A}$, RPA foci appear in a much higher frequency of cells; $~ 20$ percent (Figure 6A and B; [5]). As a means of determining the mechanisms by which the gene deletions suppress $m c m 2_{A A}$, we tested whether they also suppress the increased frequency of cells with RPA foci. We transformed a plasmid encoding GFP-tagged Rpa1 into a subset of the suppressor strains and scored each for cells with RPA foci (Figure 6B). In isolation, the gene deletions had a higher ratio of cells with RPA foci than wild type (Figure 6B) with the tof1, $\operatorname{rad} 9, \operatorname{mbp} 1, \operatorname{hrd} 1, \operatorname{ssm} 4$, sip18 and rpl8b deletions having p values less than 0.05 , reflecting the effect of these deletions on genomic stability. Despite this increase, deletion of most of the genes tested suppressed the increased frequency of RPA foci in the $m c m 2_{A A}$ strain; srs $2 \Delta$ did not $(\mathrm{p} \geq 0.05)$. Deletion of tof 1 also did not suppress $m c m 2_{A A}$. Indeed, tof $1 \Delta$ in the

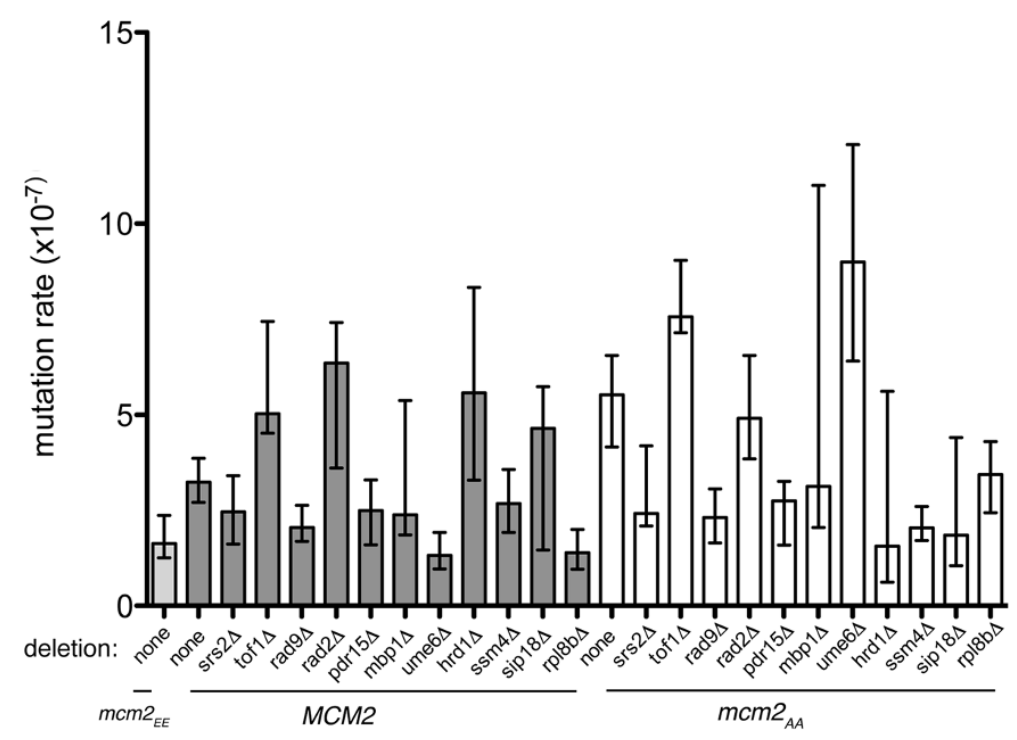

Figure 4 Mutation rates of $m \boldsymbol{c m} 2$ alleles and strains with gene deletions that suppress $m \boldsymbol{c m} 2_{A A}$. The mutation rates of $M C M 2$ and $m c m 2_{A A}$ strains with and without gene deletions that suppress $m c m 2_{A A}$ were calculated as described $[39,40]$ using the CAN1 forward mutation assay. Dark grey bars are the deletions in the MCM2 background; white bars are with $m c m 2_{A A}$ and the light grey bar (far left) is the $m c m 2_{E E}$ strain. The error bars represent the upper and lower confidence limits (95\%) of the mutation rates and were calculated from the $95 \%$ confidence limits of the median determined from the binomial distribution [40]. 


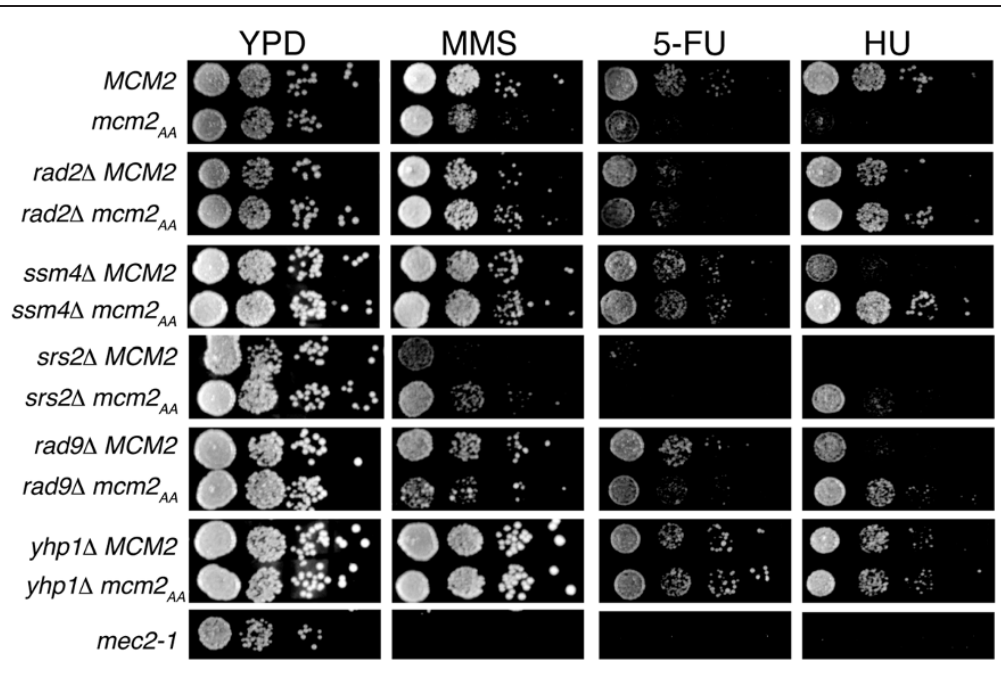

Figure 5 Sensitivity of strains containing gene deletions that suppress $m \mathrm{~cm} \mathbf{2}_{A A}$ to MMS, 5 -FU and $\mathrm{HU}$. The growth of strains containing deletions that suppress the caffeine sensitivity of $\mathrm{mcm}_{\mathrm{AA}}$ was examined on YPD with and without $0.03 \% \mathrm{MMS}, 400 \mathrm{\mu M} 5$-FU or $200 \mathrm{mM}$ HU. Tenfold serial dilutions of the strains were spotted on each of the plates and grown for the indicated times. Shown here are selected strains. The results with all of the suppressors are shown in Additional file 1.

wild type background greatly increases the frequency of cells with RPA foci. This increased frequency might be explained by the observation that disruption of the tof 1

Table 3 Phenotypes of deletions that suppress the caffeine sensitivity of $\mathbf{m c m} \mathbf{2}_{A A}$

\begin{tabular}{|c|c|c|c|c|c|c|c|c|c|}
\hline \multirow[b]{2}{*}{ mutation } & \multicolumn{4}{|c|}{ Sensitivities } & \multicolumn{5}{|c|}{ Suppression of $m c m 2_{A A}$} \\
\hline & caff & MMS & 5-FU & $\overline{\mathrm{HU}}$ & MMS & 5-FU & $\mathrm{HU}$ & $\begin{array}{l}\text { RFA } \\
\text { foci }\end{array}$ & $\begin{array}{l}\text { mutation } \\
\text { rate }\end{array}$ \\
\hline $\operatorname{srs} 2 \Delta$ & $\mathrm{R}$ & $S$ & $\mathrm{~S}$ & $\mathrm{~S}$ & - & - & + & + & + \\
\hline merls & $\mathrm{R}$ & $\mathrm{R}$ & $\mathrm{S}$ & $S$ & - & + & + & ND & ND \\
\hline tof1 $\Delta$ & $\mathrm{S}$ & $\mathrm{S}$ & $\mathrm{R}$ & $\mathrm{S}$ & + & + & $+/-$ & - & - \\
\hline $\operatorname{rad} 9 \Delta$ & $\mathrm{S}$ & $\mathrm{S}$ & $\mathrm{R}$ & $\mathrm{S}$ & - & - & + & + & + \\
\hline $\operatorname{rad} 2 \Delta$ & $\mathrm{S}$ & $\mathrm{S}$ & $\mathrm{S}$ & S & + & + & + & + & - \\
\hline pdr15 & $\mathrm{S}$ & $\mathrm{R}$ & $S$ & $\mathrm{R}$ & + & + & + & + & + \\
\hline$r d s 2 \Delta$ & $\mathrm{R}$ & $S$ & $S$ & $S$ & + & $+/-$ & + & ND & ND \\
\hline yhpls & $\mathrm{R}+$ & $\mathrm{R}+$ & $\mathrm{R}+$ & $\mathrm{R}$ & + & + & + & ND & ND \\
\hline$m b p 1 \Delta$ & $S$ & $R$ & $R$ & $S$ & + & + & + & + & - \\
\hline ume6 $\Delta$ & $\mathrm{S}$ & $\mathrm{R}$ & $\mathrm{R}$ & $\mathrm{R}$ & + & + & - & + & - \\
\hline$\overline{h r d 1 \Delta}$ & $\mathrm{S}$ & $S$ & $\mathrm{R}$ & $S$ & + & + & + & + & + \\
\hline $\operatorname{ssm} 4 \Delta$ & $\mathrm{R}+$ & $R$ & $\mathrm{R}$ & $S$ & + & + & + & + & + \\
\hline pac10s & $\mathrm{R}$ & $S$ & $S$ & $S$ & + & + & + & ND & ND \\
\hline $\operatorname{sip} 18 \Delta$ & $\mathrm{S}$ & $S$ & $\mathrm{R}$ & $\mathrm{R}+$ & + & + & + & + & + \\
\hline$r p / 8 b \Delta$ & $\mathrm{R}+$ & $S$ & $S$ & $\mathrm{~S}$ & + & + & + & + & + \\
\hline ett1 $\Delta$ & $\mathrm{R}$ & $\mathrm{R}+$ & $\mathrm{R}$ & $\mathrm{R}+$ & + & + & + & ND & ND \\
\hline
\end{tabular}

The sensitivities of the deletion strains (with wild type MCM2) to each drug is indicated by " $\mathrm{R}$ " for growth similar to wild type, " $\mathrm{S}$ " for growth slower than wild type or "R+" for better than wild type growth. For suppression of the phenotypes associated with $m \mathrm{~cm} 2_{A A}$, "-"indicates no suppression, "+" indicates suppression, " $\mathrm{E}$ " indicates epistasis and "ND" indicates that the test was not performed on that strain. ortholog in S. pombe (swi1) decouples polymerases, generating excess single stranded DNA [50-52].

\section{Discussion}

Our findings suggest a role for phosphorylation of Mcm2 by DDK in response to replicative stress. Specifically, we demonstrate that the $m c m 2_{A A}$ strain is sensitive to drugs that cause replicative stress, has an increased mutation rate and that $m c m 2_{A A}$ interacts with genes involved in the response to replicative stress. Along with our previous study showing that phosphorylation of Mcm2 at S164 and S170 slows DNA unwinding and results in enhanced DNA binding by Mcm2-7 in vitro [5], our results lead to a model in which phosphorylation of $\mathrm{Mcm} 2$ slows DNA unwinding by Mcm2-7 and/or stabilizes the replication fork as part of the proper response to replicative stress.

When a replication fork encounters DNA damage such as a base lesion or a break in the DNA strand, synthesis by the replicative polymerases at that fork halts. A series of events must then occur for replication to proceed [53]. While double stranded DNA breaks, base damage or nucleotide depletion each induce the $\mathrm{S}$ phase checkpoint, which inhibits further initiation of DNA replication and stabilizes replication forks, the form of the response differs depending on the type of perturbation [54,55]. Given the sensitivity of the $m c m 2_{A A}$ strain to MMS and 5-FU, we propose that phosphorylation of $\mathrm{Mcm} 2$ by DDK is required to stabilize replication forks in response to DNA base damage. The lack of sensitivity to phleomycin with this strain suggests that $\mathrm{Mcm} 2$ phosphorylation may not be required in response to double strand breaks. That DDK phosphorylation would trigger $\mathrm{Mcm} 2$ participation in the response to 


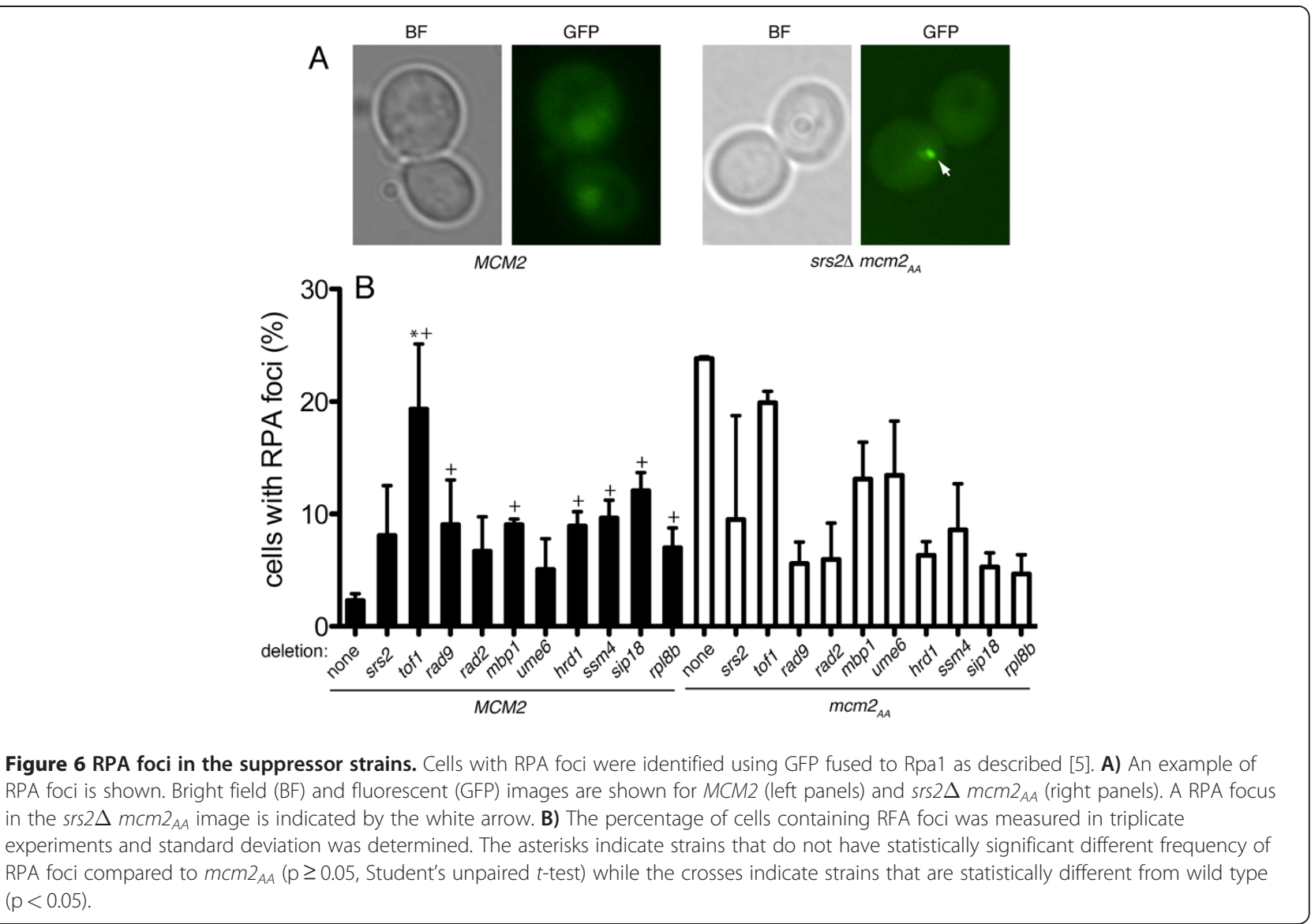

replicative stress is not surprising given DDK participates in responses to DNA damage and replicative stress and is a target of Rad53 during the $\mathrm{S}$ phase checkpoint [56-60].

\section{Genetic interactions with $\mathrm{mcm} 2_{A A}$}

The genetic interactions with $m c m 2_{A A}$ are most consistent with a requirement for $\mathrm{Mcm} 2$ phosphorylation in response to disruption of the replication fork. The effect of the suppressing deletions can be explained as either decreasing spontaneous DNA damage, which would otherwise disrupt replication forks or increasing the capacity for rescue of disrupted forks by recombination. In contrast, the deletions that result in synthetic lethal interactions increase spontaneous DNA damage, perturb the replication fork and/or are required for checkpoint responses. Mapping the interactions within the 25 genes connected to $m c m 2_{A A}$ indicates that about 15 form a network independently of $\mathrm{mcm} 2$ (Figure 7). Most of the interacting genes have roles in response to DNA damage and replicative stress. Interestingly, several of the genes interact with $C D C 7$ and $D B F 4$, the genes encoding the two components of DDK (Figure 7).

Of the 25 genetic interactions with $\mathrm{mcm} 2$ that we isolated, only one was previously identified. Deletion of $\mathrm{rad} 9$ has a synthetic lethal interaction with $m c m 2-1$, a temperature sensitive allele [63]. Interestingly, here rad9 $\Delta$ suppresses some, but not all, of the phenotypes associated with $m c m 2_{A A}$. The difference may result from allele specificity; $m c m 2-1$ is expected to affect Mcm2's essential role in Mcm2-7 function whereas the $m c m 2_{A A}$ allele affects $\mathrm{Mcm} 2$ activity in response to replicative stress.

Deletion of three genes, HRD1, SSM4 and PAC10 whose products are involved in targeting misfolded proteins for degradation $[64,65]$, suppress $m c m 2_{A A}$. One possible mechanism for these suppressors is that misfolded proteins induce the unfolded protein response (UPR) which in turn provides protection against reactive oxygen species which can damage DNA [66]. However, the mechanism of suppression is more complex since $m c m 2_{A A}$ is still sensitive to caffeine or MMS in the presence of tunicamycin, which induces the UPR (data not shown).

\section{Regulating helicase progression in response to replicative stress}

We propose that phosphorylation of $\mathrm{Mcm} 2$ by DDK is required in response to replicative stress. This role is not recognition of damage or replication fork collapse since the $S$ phase checkpoint is intact at least up to Rad53 phosphorylation. Furthermore, $m c m 2_{A A}$ has synthetic lethal 


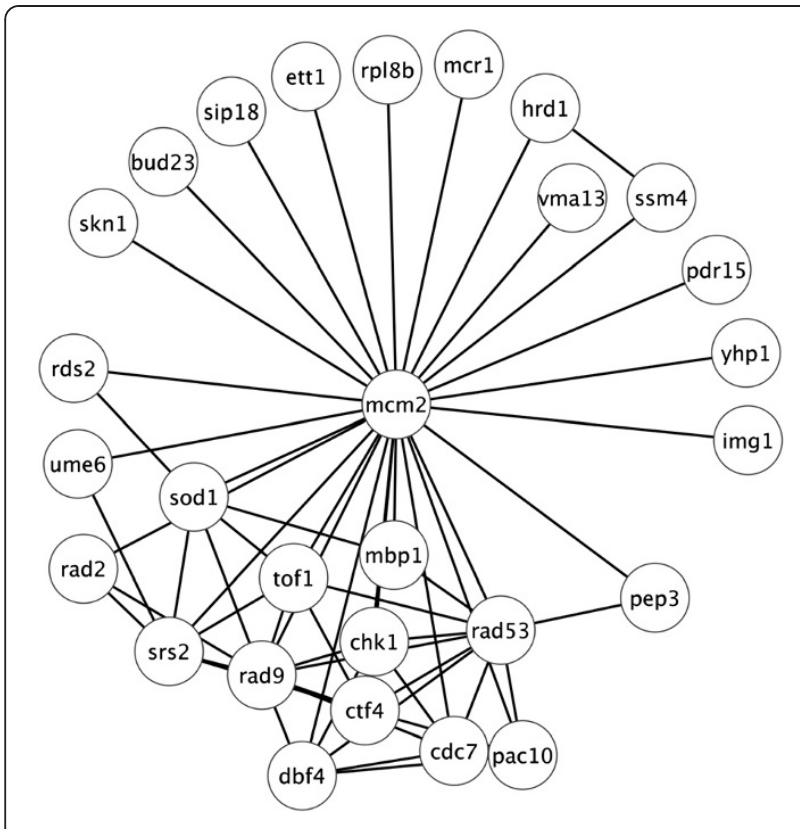

Figure $\mathbf{7}$ Interactions amongst $\mathbf{m c m} \mathbf{2}_{A A}$ interactors. A map of interactions amongst the genes connected with $m c m 2_{A A}$ was generated using Cytoscape 2.8.2 [61,62]. Physical and genetic interactions annotated on SGD [37] were used to generate the map. Also included are interactions within this group with CDC7 and DBF4, the genes encoding the components of DDK.

interactions with $\operatorname{ch} k 1 \Delta$ and $m e c 2-1$, mutations in the genes encoding the checkpoint effector kinases in yeast [2,67-69]. Synthetic lethal interactions often indicate function of the interacting genes in parallel pathways. Based on the previously observed biochemical activities of Mcm2-7 with DDK-phosphorylated or phosphomimetic Mcm2 [5], we surmise that phosphorylation of $\mathrm{Mcm} 2$ may stabilize Mcm2-7 on DNA and/or slow the helicase. DNA helicases are predicted to contact DNA through the sugar phosphate backbone, not the bases [70]. Therefore, unlike replicative polymerases that stall at sites with missing or damaged bases, the helicase will continue unwinding DNA and may decouple from the polymerase. Indeed single stranded DNA generated by decoupling of helicase from the replicative polymerases is proposed to generate a checkpoint response [71-77]. Decoupling appears to be regulated so that the helicase does not advance too far from the rest of the replisome, leading to complete disassembly of replication forks [78]. One role for Mcm2 phosphorylation may be to slow the helicase so that it does not proceed too far ahead of the replicative polymerases. In this model, the accumulation of RPA foci in the $m c m 2_{A A}$ strain is not due to spontaneous decoupling of the helicase from polymerase but rather is due to DNA damage triggering decoupling of the helicase.

The idea that replicative stress triggers a requirement for Mcm2 phosphorylation by DDK is supported by the synthetic lethal interaction of $m c m 2_{A A}$ with deletions that lead to genomic instability. Examples include $c t f 4 \Delta$ and img1 4 . Ctf4 is involved in sister chromatid cohesion and is integral to the RPC [16,36,79-81]. Deletion of img1 leads to loss of functional mitochondria which in turn leads to genomic instability $[34,35]$. Increased DNA damage is also noted in strains with increased levels of ROS, such as $\operatorname{sod} 1 \Delta[33]$ and vma13 $[82,83]$, both of which are synthetically lethal with $m c m 2_{A A}$. Rad9 is important for transmission of checkpoint signalling with deletion of rad9 resulting in cells that fail to arrest in response to DNA damage $[84,85]$. The increased homologous recombination noted with srs $2 \Delta$, $\operatorname{rad} 2 \Delta$ and ume6 $\Delta$ may suppress $m c m 2_{A A}$ by providing a means to resolve stalled replication forks [45,86-88]. Finally, the model that Mcm2 phosphorylation may be required to slow replication forks is supported by the ability of tof $1 \Delta$ to suppress defects of $m c m 2_{A A}$ only in the presence of genotoxic agents (tof $1 \Delta$ does not suppress the increased mutation rate or increased frequency of RPA foci). Lack of Tof1, a member of the RPC, slows replication forks [43], however this occurs only in the presence of replicative stress, such as seen in the presence of genotoxic agents [42].

\section{Conclusions}

Phosphorylation of $\mathrm{Mcm} 2$ by DDK is required for the proper response to replicative stress, but not to induce a checkpoint. This phosphorylation event likely slows the Mcm2-7 helicase and/or stabilizes replication forks. In the absence of $\mathrm{Mcm} 2$ phosphorylation, the mutation rate is increased.

\section{Methods}

\section{Materials}

Caffeine, HU and MMS were purchased from Sigma Aldrich (99\% purity); geneticin (G418) from either United States Biological or Santa Cruz Biotechnology; phleomycin from Santa Cruz Biotechnology; 5-FU from Nutritional Biochemicals Corp. YPD is $1 \%$ yeast extract, $2 \%$ peptone and 2\% D-glucose. The yeast strains MDY167 (MCM2-URA3), MDY169 ( $\left.m c m 2_{A A}-U R A 3\right)$ and MDY191 ( $\left.m c m 2_{E E}-U R A 3\right)$ are described in Stead et al. [5] and the mec2-1 strain in [24].

\section{Synthetic lethal screen}

The genetic screens were a modification of SGA analysis [89]. Manipulation of the gene arrays was performed manually using a 3.18-mm 48-pinner tool (V\&P Scientific, San Diego, CA). Screens were performed using MDY169

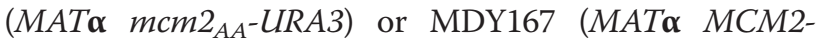
$U R A 3$ [5]) mated with the haploid yeast Magic Marker deletion collection generated from the diploid strain collection (Open Biosystems; [90]). Diploids were selected and then sporulated before selecting haploid cells that 
contained a gene deletion $\left(\operatorname{Kan}^{\mathrm{R}}\right)$ and $m c m 2_{A A}$ or $M C M 2$ $\left(\mathrm{Ura}^{+}\right)$. Plates were scanned on a HP Scanjet 3970 and colony size was compared using ImageQuant TL (GE Healthcare). A synthetic lethal or slow growth (SSL) phenotype was assigned if the colony size was smaller in the $m c m 2_{A A}$ cross than in the MCM2 cross. A false positive rate (growth when the gene deletion is reported as lethal in SGD [37]) was roughly $5 \%$ and is similar to false positive rates reported previously [89]. From this initial screen, we compiled a list of 234 genes that had SSL interactions with $m c m 2_{A A}$. Next, the candidates were retested from the mating step in triplicate. Forty-one SSL interactions were identified in this step. The deletions were then re-tested for genetic interactions with $m c m 2_{A A}$ by re-mating followed by tetrad dissection. SSL interactions were verified if Ura + and $\mathrm{G} 418^{\mathrm{R}}$ spore progeny were never recovered or grew slowly relative to the corresponding single mutations.

\section{Screen for suppressors of caffeine sensitivity}

A screen to isolate deletions that suppress the caffeine sensitivity of $m c m 2_{A A}$ was performed by pinning the haploid $m c m 2_{A A}$ strains that also contained a gene deletion (generated as described above) to YPD containing $15 \mathrm{mM}$ caffeine. The plates were incubated at $30^{\circ} \mathrm{C}$ for 4 days, imaged and then quantified. A spot was determined to contain a deletion that suppresses $m c m 2_{A A}$ when its size was larger than the spot size of the $m c m 2_{A A}$ strain. The deletion of 369 genes suppressed the growth defect of $m c m 2_{A A}$ in caffeine in the initial screen. These candidate deletions were re-mated in triplicate, haploids generated and retested for sensitivity to caffeine, resulting in 86 strains containing a deletion that potentially suppresses $m c m 2_{A A}$. The 86 were strains re-mated, sporulated and tetrads dissected. A G418 resistant, Ura + colony was identified, grown to saturation and 10-fold serial dilutions were spotted onto YPD with and without $15 \mathrm{mM}$ caffeine.

\section{Mutation rate assay}

The forward mutation assay was performed as described in [91]. Briefly, at least 20 colonies were inoculated into $10 \times 1 \mathrm{ml} \mathrm{YPD}$ and the cultures grown overnight at $30^{\circ} \mathrm{C}$ to $1-2 \times 10^{8}$ cells $/ \mathrm{ml}$. Each culture was diluted to approximately 200 cells $/ 1 \mathrm{ml} \mathrm{YPD}$ and grown to $1-2 \times 10^{8}$ cells $/ \mathrm{ml}$. Cells were then plated $\left(\sim 10^{7}\right)$ on CM-Arg plates containing $25 \mu \mathrm{g}$ canavanine $/ \mathrm{ml}$ and appropriate dilutions were made before plating on YPD. Colonies on each plate were counted to determine the number of canavanine resistant cells per $10^{7}$ cells. The mutation rate was calculated using the method of the median $[39,40]$.

\section{Additional file}

Additional file 1: Stead et al., Supplemental Data.

\section{Acknowledgements}

This work was supported by a Canadian Institutes of Health Research Operating Grant (MOP 68926) to MJD and by funding from Schulich School of Medicine \& Dentistry. BES was supported by a Natural Science and Engineering Research Council PGS-D fellowship.

\section{Authors' contributions}

BES carried out the screens and drug assays with assistance from CJB and MKS and wrote a draft of the manuscript. MJD did the microscopy, mutation assays, Rad53 blot and edited the manuscript with CJB. All authors read and approved the final manuscript.

Received: 29 January 2012 Accepted: 7 May 2012

Published: 7 May 2012

\section{References}

1. Bell SP, Dutta A: DNA replication in eukaryotic cells. Annu Rev Biochem 2002, 71:333-374

2. Segurado $M$, Tercero JA: The S-phase checkpoint: targeting the replication fork. Biol Cell 2009, 101:617-627.

3. Randell JC, Fan A, Chan C, Francis LI, Heller RC, Galani K, Bell SP: Mec1 is one of multiple kinases that prime the $\mathrm{Mcm} 2-7$ helicase for phosphorylation by Cdc7. Mol Cell 2010, 40:353-363.

4. Sheu YJ, Stillman B: Cdc7-Dbf4 phosphorylates MCM proteins via a docking site-mediated mechanism to promote $\mathrm{S}$ phase progression. $\mathrm{Mo}$ Cell 2006, 24:101-113.

5. Stead BE, Brandl CJ, Davey MJ: Phosphorylation of Mcm2 modulates Mcm2-7 activity and affects the cell's response to DNA damage. Nucleic Acids Res 2011, 39:6998-7008

6. Cortez D, Glick G, Elledge SJ: Minichromosome maintenance proteins are direct targets of the ATM and ATR checkpoint kinases. Proc Natl Acad Sci US A 2004, 101:10078-10083.

7. Montagnoli A, Valsasina B, Brotherton D, Troiani S, Rainoldi S, Tenca P, Molinari A, Santocanale C: Identification of $\mathrm{Mcm} 2$ phosphorylation sites by S-phase-regulating kinases. J Biol Chem 2006, 281:10281-10290.

8. Yoo HY, Shevchenko A, Dunphy WG: Mcm2 is a direct substrate of ATM and ATR during DNA damage and DNA replication checkpoint responses. J Biol Chem 2004, 279:53353-53364.

9. Bochman ML, Schwacha A: The Mcm complex: unwinding the mechanism of a replicative helicase. Microbiol Mol Biol Rev 2009, 73:652-683.

10. Forsburg SL: Eukaryotic MCM proteins: beyond replication initiation. Microbiol Mol Biol Rev 2004, 68:109-131.

11. Moyer SE, Lewis PW, Botchan MR: Isolation of the Cdc45/Mcm2-7/GINS (CMG) complex, a candidate for the eukaryotic DNA replication fork helicase. Proc Natl Acad Sci U S A 2006, 103:10236-10241.

12. Pacek M, Tutter AV, Kubota Y, Takisawa H, Walter JC: Localization of MCM27, Cdc45, and GINS to the site of DNA unwinding during eukaryotic DNA replication. Mol Cell 2006, 21:581-587

13. Sheu YJ, Stillman B: The Dbf4-Cdc7 kinase promotes $S$ phase by alleviating an inhibitory activity in Mcm4. Nature 2010, 463:113-117.

14. Ishimi $Y$, Komamura-Kohno $Y$ : Phosphorylation of Mcm4 at specific sites by cyclin-dependent kinase leads to loss of Mcm4,6,7 helicase activity. $J$ Biol Chem 2001, 276:34428-34433.

15. Costa A, Ilves I, Tamberg N, Petojevic T, Nogales E, Botchan MR, Berger JM: The structural basis for MCM2-7 helicase activation by GINS and Cdc45. Nat Struct Mol Biol 2011, 18:471-477.

16. Gambus A, Jones RC, Sanchez-Diaz A, Kanemaki M, van Deursen F, Edmondson RD, Labib K: GINS maintains association of Cdc45 with MCM in replisome progression complexes at eukaryotic DNA replication forks. Nat Cell Biol 2006, 8:358-366.

17. Ilves I, Petojevic T, Pesavento JJ, Botchan MR: Activation of the MCM2-7 helicase by association with Cdc45 and GINS proteins. Mol Cell 2010, 37:247-258.

18. Hall-Jackson CA, Cross DA, Morrice N, Smythe C: ATR is a caffeine-sensitive, DNA-activated protein kinase with a substrate specificity distinct from DNA-PK. Oncogene 1999, 18:6707-6713.

19. Kuranda K, Leberre V, Sokol S, Palamarczyk G, Francois J: Investigating the caffeine effects in the yeast Saccharomyces cerevisiae brings new insights into the connection between TOR, PKC and Ras/cAMP signalling pathways. Mol Microbiol 2006, 61:1147-1166.

20. Reinke A, Chen JC, Aronova S, Powers T: Caffeine targets TOR complex I and provides evidence for a regulatory link between the FRB and kinase domains of Tor1p. J Biol Chem 2006, 281:31616-31626. 
21. Wanke V, Cameroni E, Uotila A, Piccolis M, Urban J, Loewith R, De Virgilio C: Caffeine extends yeast lifespan by targeting TORC1. Mol Microbio/ 2008, 69:277-285

22. Saiardi A, Resnick AC, Snowman AM, Wendland B, Snyder SH: Inositol pyrophosphates regulate cell death and telomere length through phosphoinositide 3-kinase-related protein kinases. Proc Natl Acad Sci U S A 2005, 102:1911-1914.

23. Tercero JA, Diffley JF: Regulation of DNA replication fork progression through damaged DNA by the Mec1/Rad53 checkpoint. Nature 2001, 412:553-557.

24. Weinert TA, Kiser GL, Hartwell LH: Mitotic checkpoint genes in budding yeast and the dependence of mitosis on DNA replication and repair. Genes Dev 1994, 8:652-665.

25. Beranek DT: Distribution of methyl and ethyl adducts following alkylation with monofunctional alkylating agents. Mutat Res 1990, 231:11-30.

26. Seiple L, Jaruga P, Dizdaroglu M, Stivers JT: Linking uracil base excision repair and 5-fluorouracil toxicity in yeast. Nucleic Acids Res 2006, 34:140-151.

27. Moore CW: Cleavage of cellular and extracellular Saccharomyces cerevisiae DNA by bleomycin and phleomycin. Cancer Res 1989, 49:6935-6940.

28. Cobb JA, Bjergbaek L, Shimada K, Frei C, Gasser SM: DNA polymerase stabilization at stalled replication forks requires Mec1 and the RecQ helicase Sgs1. EMBO J 2003, 22:4325-4336.

29. Santocanale C, Diffley JF: A Mec1- and Rad53-dependent checkpoint controls late-firing origins of DNA replication. Nature 1998, 395:615-618.

30. Bernstein KA, Shor E, Sunjevaric I, Fumasoni M, Burgess RC, Foiani M, Branzei $D$, Rothstein R: Sgs1 function in the repair of DNA replication intermediates is separable from its role in homologous recombinational repair. EMBO J 2009, 28:915-925.

31. Clausing E, Mayer A, Chanarat S, Muller B, Germann SM, Cramer P, Lisby M, Strasser K: The transcription elongation factor Bur1-Bur2 interacts with replication protein $A$ and maintains genome stability during replication stress. J Biol Chem 2010, 285:41665-41674

32. Wu X, Shell SM, Zou Y: Interaction and colocalization of Rad9/Rad1/Hus1 checkpoint complex with replication protein $\mathrm{A}$ in human cells. Oncogene 2005, 24:4728-4735

33. Gralla EB, Valentine JS: Null mutants of Saccharomyces cerevisiae $\mathrm{Cu}, \mathrm{Zn}$ superoxide dismutase: characterization and spontaneous mutation rates. J Bacteriol 1991, 173:5918-5920

34. Coppee JY, Rieger KJ, Kaniak A, di Rago JP, Groudinsky O, Slonimski PP: PetCR46, a gene which is essential for respiration and integrity of the mitochondrial genome. Yeast 1996, 12:577-582.

35. Rasmussen AK, Chatterjee A, Rasmussen LJ, Singh KK: Mitochondriamediated nuclear mutator phenotype in Saccharomyces cerevisiae. Nucleic Acids Res 2003, 31:3909-3917.

36. Kouprina N, Kroll E, Bannikov V, Bliskovsky V, Gizatullin R, Kirillov A, Shestopalov B, Zakharyev V, Hieter P, Spencer F, et al: CTF4 (CHL15) mutants exhibit defective DNA metabolism in the yeast Saccharomyces cerevisiae. Mol Cell Biol 1992, 12:5736-5747.

37. Saccharomyces Genome Database.www.yeastgenome.org

38. Foiani M, Liberi G, Piatti S, Plevani P: Saccharomyces cerevisiae as a model system to study DNA replication. In Practical Approach Edited by Cotterill S. Edited by Eukaryotic DNA, Replication A. Oxford, UK: Oxford University Press; 1999:185-200

39. Lea $D$, Coulson $C$ : The distribution of the numbers of mutants in bacterial populations. J Genet 1949, 49:264-285.

40. Foster PL: Methods for determining spontaneous mutation rates. Methods Enzymol 2006, 409:195-213.

41. Bairwa NK, Mohanty BK, Stamenova R, Curcio MJ, Bastia D: The intra-S phase checkpoint protein Tof1 collaborates with the helicase Rrm3 and the F-box protein Dia2 to maintain genome stability in Saccharomyces cerevisiae. J Biol Chem 2011, 286:2445-2454

42. Hodgson B, Calzada A, Labib K: Mrc1 and Tof1 regulate DNA replication forks in different ways during normal S phase. Mol Biol Cell 2007, 18:3894-3902.

43. Tourriere H, Versini G, Cordon-Preciado V, Alabert C, Pasero P: Mrc1 and Tof1 promote replication fork progression and recovery independently of Rad53. Mol Cell 2005, 19:699-706.

44. Habraken Y, Sung P, Prakash S, Prakash L: Transcription factor TFIIH and DNA endonuclease Rad2 constitute yeast nucleotide excision repair factor 3: implications for nucleotide excision repair and Cockayne syndrome. Proc Natl Acad Sci U S A 1996, 93:10718-10722.

45. Sweet DH, Jang YK, Sancar GB: Role of UME6 in transcriptional regulation of a DNA repair gene in Saccharomyces cerevisiae. Mol Cell Biol 1997, 17:6223-6235

46. Jani NM, Lopes JM: Transcription regulation of the Saccharomyces cerevisiae PIS1 gene by inositol and the pleiotropic regulator, Ume6p. Mol Microbiol 2008, 70:1529-1539.

47. Steinfeld I, Shamir R, Kupiec M: A genome-wide analysis in Saccharomyces cerevisiae demonstrates the influence of chromatin modifiers on transcription. Nat Genet 2007, 39:303-309.

48. Williams RM, Primig M, Washburn BK, Winzeler EA, Bellis M, Sarrauste de Menthiere C, Davis RW, Esposito RE: The Ume6 regulon coordinates metabolic and meiotic gene expression in yeast. Proc Natl Acad Sci U S A 2002, 99:13431-13436.

49. Pramila T, Miles S, GuhaThakurta D, Jemiolo D, Breeden LL: Conserved homeodomain proteins interact with MADS box protein $\mathrm{Mcm} 1$ to restrict ECB-dependent transcription to the M/G1 phase of the cell cycle. Genes Dev 2002, 16:3034-3045.

50. Sommariva E, Pellny TK, Karahan N, Kumar S, Huberman JA, Dalgaard JZ: Schizosaccharomyces pombe Swi1, Swi3, and Hsk1 are components of a novel S-phase response pathway to alkylation damage. Mol Cell Biol 2005, 25:2770-2784

51. Vengrova S, Dalgaard JZ: RNase-sensitive DNA modification(s) initiates $S$. pombe mating-type switching. Genes Dev 2004, 18:794-804.

52. Noguchi E, Noguchi C, McDonald WH, Yates JR 3rd, Russell P: Swi1 and Swi3 are components of a replication fork protection complex in fission yeast. Mol Cell Biol 2004, 24:8342-8355.

53. McGlynn P, Lloyd RG: Recombinational repair and restart of damaged replication forks. Nat Rev Mol Cell Biol 2002, 3:859-870

54. Chang M, Bellaoui M, Boone C, Brown GW: A genome-wide screen fo methyl methanesulfonate-sensitive mutants reveals genes required for $S$ phase progression in the presence of DNA damage. Proc Natl Acad Sci U S A 2002, 99:16934-16939.

55. Putnam CD, Jaehnig EJ, Kolodner RD: Perspectives on the DNA damage and replication checkpoint responses in Saccharomyces cerevisiae. DNA Repair 2009, 8:974-982.

56. Duncker BP, Brown GW: Cdc7 kinases (DDKs) and checkpoint responses: lessons from two yeasts. Mutat Res 2003, 532:21-27.

57. Gabrielse C, Miller CT, McConnell KH, DeWard A, Fox CA, Weinreich M: A Dbf4p BRCA1 C-terminal-like domain required for the response to replication fork arrest in budding yeast. Genetics 2006, 173:541-555.

58. Ogi H, Wang CZ, Nakai W, Kawasaki Y, Masumoto H: The role of the Saccharomyces cerevisiae Cdc7-Dbf4 complex in the replication checkpoint. Gene 2008, 414:32-40.

59. Pessoa-Brandao L, Sclafani RA: CDC7/DBF4 functions in the translesion synthesis branch of the RAD6 epistasis group in Saccharomyces cerevisiae. Genetics 2004, 167:1597-1610.

60. Weinreich M, Stillman B: Cdc7p-Dbf4p kinase binds to chromatin during S phase and is regulated by both the APC and the RAD53 checkpoint pathway. EMBO J 1999, 18:5334-5346.

61. Cline MS, Smoot M, Cerami E, Kuchinsky A, Landys N, Workman C, Christmas R, Avila-Campilo I, Creech M, Gross B, et al: Integration of biological networks and gene expression data using Cytoscape. Nat Protoc 2007, 2:2366-2382.

62. Smoot ME, Ono K, Ruscheinski J, Wang PL, Ideker T: Cytoscape 2.8: new features for data integration and network visualization. Bioinformatics 2011, 27:431-432.

63. Costanzo M, Baryshnikova A, Bellay J, Kim Y, Spear ED, Sevier CS, Ding H, Koh JL, Toufighi K, Mostafavi S, et al: The genetic landscape of a cell. Science 2010, 327:425-431.

64. Alvarez P, Smith A, Fleming J, Solomon F: Modulation of tubulin polypeptide ratios by the yeast protein Pac10p. Genetics 1998, 149:857-864.

65. Ismail N, Ng DTW: Have you HRD? Understanding ERAD Is DOAble! Cell 2006, 126:237-239.

66. Gregersen N, Bross P: Protein misfolding and cellular stress: an overview. Meth Mol Biol 2010, 648:3-23

67. Chen $Y$, Sanchez $Y$ : Chk1 in the DNA damage response: conserved roles from yeasts to mammals. DNA Repair (Amst) 2004, 3:1025-1032. 
68. Liu Y, Vidanes G, Lin YC, Mori S, Siede W: Characterization of a Saccharomyces cerevisiae homologue of Schizosaccharomyces pombe Chk1 involved in DNA-damage-induced M-phase arrest. Mol Gen Genet 2000, 262:1132-1146.

69. Rouse J, Jackson SP: Interfaces between the detection, signaling, and repair of DNA damage. Science 2002, 297:547-551.

70. Enemark EJ, Joshua-Tor L: Mechanism of DNA translocation in a replicative hexameric helicase. Nature 2006, 442:270-275.

71. Byun TS, Pacek M, Yee MC, Walter JC, Cimprich KA: Functional uncoupling of MCM helicase and DNA polymerase activities activates the ATRdependent checkpoint. Genes Dev 2005, 19:1040-1052.

72. Feng W, Collingwood D, Boeck ME, Fox LA, Alvino GM, Fangman WL, Raghuraman MK, Brewer BJ: Genomic mapping of single-stranded DNA in hydroxyurea-challenged yeasts identifies origins of replication. Nat Cell Biol 2006, 8:148-155.

73. Lucca C, Vanoli F, Cotta-Ramusino C, Pellicioli A, Liberi G, Haber J, Foiani M: Checkpoint-mediated control of replisome-fork association and signalling in response to replication pausing. Oncogene 2004, 23:1206-1213.

74. Nedelcheva MN, Roguev A, Dolapchiev LB, Shevchenko A, Taskov HB, Stewart AF, Stoynov SS: Uncoupling of unwinding from DNA synthesis implies regulation of MCM helicase by Tof1/Mrc1/Csm3 checkpoint complex. J Mol Biol 2005, 347:509-521.

75. Sogo JM, Lopes M, Foiani M: Fork reversal and ssDNA accumulation at stalled replication forks owing to checkpoint defects. Science 2002, 297:599-602.

76. You Z, Kong L, Newport J: The role of single-stranded DNA and polymerase alpha in establishing the ATR, Hus1 DNA replication checkpoint. J Biol Chem 2002, 277:27088-27093.

77. Zou L, Elledge SJ: Sensing DNA damage through ATRIP recognition of RPA-ssDNA complexes. Science 2003, 300:1542-1548.

78. Nitani N, Yadani C, Yabuuchi H, Masukata H, Nakagawa T: Mcm4 C-terminal domain of MCM helicase prevents excessive formation of singlestranded DNA at stalled replication forks. Proc Natl Acad Sci 2008, 105:12973-12978.

79. Hanna JS, Kroll ES, Lundblad V, Spencer FA: Saccharomyces cerevisiae CTF18 and CTF4 are required for sister chromatid cohesion. Mol Cell Biol 2001, 21:3144-3158.

80. Miles J, Formosa T: Evidence that POB1, a Saccharomyces cerevisiae protein that binds to DNA polymerase alpha, acts in DNA metabolism in vivo. Mol Cell Biol 1992, 12:5724-5735.

81. Gambus A, van Deursen F, Polychronopoulos D, Foltman M, Jones RC, Edmondson RD, Calzada A, Labib K: A key role for Ctf4 in coupling the MCM2-7 helicase to DNA polymerase alpha within the eukaryotic replisome. EMBO J 2009, 28:2992-3004.

82. Ando A, Nakamura T, Murata Y, Takagi H, Shima J: Identification and classification of genes required for tolerance to freeze-thaw stress revealed by genome-wide screening of Saccharomyces cerevisiae deletion strains. FEMS Yeast Res 2007, 7:244-253.

83. Dudley AM, Janse DM, Tanay A, Shamir R, Church GM: A global view of pleiotropy and phenotypically derived gene function in yeast. Mo/ Syst Biol 2005, 1(2005):0001.

84. Paulovich AG, Margulies RU, Garvik BM, Hartwell LH: RAD9, RAD17, and RAD24 are required for S phase regulation in Saccharomyces cerevisiae in response to DNA damage. Genetics 1997, 145:45-62.

85. Toh GW, Lowndes NF: Role of the Saccharomyces cerevisiae Rad9 protein in sensing and responding to DNA damage. Biochem Soc Trans 2003, 31:242-246.

86. Krejci L, Van Komen S, Li Y, Villemain J, Reddy MS, Klein H, Ellenberger T, Sung P: DNA helicase Srs2 disrupts the Rad51 presynaptic filament. Nature 2003, 423:305-309.

87. Veaute $X$, Jeusset J, Soustelle C, Kowalczykowski SC, Le Cam E, Fabre F: The Srs2 helicase prevents recombination by disrupting Rad51 nucleoprotein filaments. Nature 2003, 423:309-312.

88. Howlett NG, Schiestl RH: Nucleotide excision repair deficiency causes elevated levels of chromosome gain in Saccharomyces cerevisiae. DNA Repair 2004, 3:127-134.

89. Tong A, Boone C: Synthetic Genetic Array (SGA) Analysis in Saccharomyces cerevisiae, Methods in Molecular Biology, Volume 313. Second Editionth edition. Totowa, NJ, U. S. A: The Humana Press, Inc; 2005:171-192. Yeast Protocols.
90. Pan X, Yuan DS, Xiang D, Wang X, Sookhai-Mahadeo S, Bader JS, Hieter P, Spencer $F$, Boeke JD: A robust toolkit for functional profiling of the yeast genome. Mol Cell 2004, 16:487-496.

91. Huang ME, Rio AG, Nicolas A, Kolodner RD: A genomewide screen in Saccharomyces cerevisiae for genes that suppress the accumulation of mutations. Proc Natl Acad Sci U S A 2003, 100:11529-11534.

doi:10.1186/1471-2156-13-36

Cite this article as: Stead et al:: Mcm2 phosphorylation and the response to replicative stress. BMC Genetics 2012 13:36.

\section{Submit your next manuscript to BioMed Central and take full advantage of:}

- Convenient online submission

- Thorough peer review

- No space constraints or color figure charges

- Immediate publication on acceptance

- Inclusion in PubMed, CAS, Scopus and Google Scholar

- Research which is freely available for redistribution 